\title{
Advanced Control Rooms and Crew Performance Issues: Implications for Human Reliability*
}

\author{
John M. O'Hara and Robert E. Hall \\ Brookhaven National Laboratory \\ Uf ‘on, New York 11973
}

BNI, -NUREG --46825

DE92 004986

\begin{abstract}
Recent trends in advanced control room (ACR) design are considered with respect to their impact on human performance. It is concluded that potentially negative influences exist, however, a variety of factors make it difficult to model, analyze, and quantify these effects for buman reliability analyses (HRAs).
\end{abstract}

\section{INTRODUCTION}

Reports of nuclear industry surveys on the use and intended applications of advanced instrumentation and controls both internationally[1] and in North America[2] indicate that the industry is rapidly developing advanced technology and that it is being applied in nuclear plants. Whiis the introduction of advanced technology has been slower in the United States, when compared with countries such as Canada, France, and Japan, its incorporation into U.S. control rooms is inevitable.

There are three important trends in the evolution of ACRs. The first trend is toward increased automation. With increased application of digital control technology comes an enhanced ability to automate tasks traditionally performed by an operator. Second, there is a trend toward the development of compact, computer-based workstations as the locus of control room operations. The third trend is the development of intelligent operator aids based on expert systems and other artificial intelligence-based technologies. These applications include aids for alarm processing, diagnostics, accident management, piant monitoring, and procedure tracking.

Related to ACR evolution is the wide range of technological approaches to human-system interfaces (HSI). In part, this is due to the tremendous flexibility offered by software-driven interfaces to provide for alternatives data display and control. The options for display not only expand the hardware media choices (from computer-driven displays which mimic conventional gauges and meters to video display units to computerdriven large screen displays) but also the formats with which to display data are nearly infinite (e.g., lists, tables, flow charts, graphs, iconic graphics, speech, etc.). Operator input to the system has seen similar expansion in diversity including, for example, conventional controls, miniature controls, touch screens, mice, joy sticks, light pens, and voice controls. With the advent of interactive graphic displays, the traditional distinction between controls and displays becomes blurred.
For example, an operator may open a valve or start a pumpy using "soft" controls such as by touching the icnn of the desired component on a computer graphic mimic of the system. In additior, data processing and integration are more significant in ACl.s providing the operator with bigher-level displays.

Developments in control room technology have significant implications for plant safety in that they will affect the role of operators in the plant, what they will be required to know about the plant and plant systems, and the ways in which they interact with them. The crew team performance and operator interaction will be affected. In addition, it is quite likely that the introduction of advanced technology will significantly affect operator selection, training, and examination requirements. Generally, the introsluction of advanced technology is viewed as having great promise to improve the safe operation of nuclear power plants (NPPs). Advanced iechnology, however, does not a priori enhance crew performance and reliability. In order to ensure the appropritte application of technology to support operator performance and piacit safaty, it is important to evaluate the introduction of new technologies in terms of their potential negative as well as positive effects. The purpose of this pap $r$ is $\%$, examine the trends in ACR technology with respect io how invarating crew performance and human reliability may be impacied.

\section{HUMAN PERFORMANCE ISSUES}

Despite the rapidly increasing utilization of advanced HSI technology in complex, high-reliability systems such as NPPs and civilian aircraft, there is broad consensus that the knowledge-base for understanding the effects of this technology on human performance and system safety is in need of further research $[4,5]$. The operating environment associated with advanced systems is very different from that of a conventional control room. With the increased use of computer-based interfaces, cognitive and human information processing issues are emerging as more significant than the physical and ergonomic considerations which dominated the design of conventional HSis. While these issues have been recognized for a long time, their full implications for human performavice and system safety have only recently begun to be addressed in research, and there is not a long history of operational experience upon which to draw. While the overall state-ofknowledge is limited, some issues can be identified from the available research and experience with advanced systems.

\footnotetext{
*Work sponsored under the auspices of the U.S. Nuclear Regulatory Commission.
}

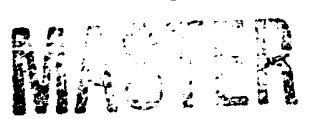




\section{DISCLAIMER}

This report was prepared as an account of work sponsored by an agency of the United Siates Government. Neither the United States Government nor any agency thereof, nor any of their employees, makes any warranty, express or implied, or assumes any legal liability or responsibility for the accuracy, completeness, or usefulness of any information, apparatus, product, or process disclosed, or represents that its use would not infringe privately owned rights. Reference herein to any specific commercial product, process, or service by trade name, trademark, manufacturer, or otherwise does not necessarily constitute or imply its endorsement, recommendation, or favoring by the United States Government or any agency thereof. The views and opinions of authors expressed herein do not necessarily state or reflect those of the United States Government or any agency thereof. 
Human performance theories typically consider errors as one indicator of performance along with other measures, such as time, accuracy, and workload. However, error has special significance in the HRA domain due to its incorporation and quantification in risk models. Therefore, within the context of the present discussion, human errors are distinguishea from other aspects of human performance. With respect to understanding the effects of ACRs on human performance, error, and safety, it is useful to consider the error event (whether omission or commission) to be the end product of an information processing sequence. Then the focus of ACR effects can be directed to the effect on errors themselves and on the information processing "precursors" that give rise to them. In the discussion that follows, precursors are considered first with respect to the ACR trends identified above: automation and HSI design. A discussion of error mechanisms is provided at the end of the Section.

One of the inajor trends in ACR design is an increase in automation of tasks traditionally performed by the operator. Increases in automation result in a shift of the operator's function in the system from that of a direct manual controller to a supervisory controller and system monitor who is largely removed from direct control. This type of role change is typically viewed as positive from a reliability standpoint, since the human operator is considered one of the more unpredictable components in the system. It is generally presumed that automation will enhance overall system reliability by removing or reducing the need for human action. The operator's performance in the system is believed to be improved by freeing him from tasks which are routine, tedious, physically demanding, or difficult. Thus, the operator can better concentrate on supervising the overall performar 2 and safety of the system.

Historically, functions have been allocated to automated systems based largely on the capability of available technology to reliably and safely execute the function. This allovation of function strategy does not consider whether a function should be automated with respect to the human operator's ability to perform as an integral part of the overall system. The operator becomes the default controller handling those functions/tasks that can not be satisfactorily automated. This strategy may leave the operators with an ill-defined role in the system and a poorly organized set of tasks. The central problem with poor allocation of function (with respect to the operator's ability to properly supervise the system and assume control when needed) is the erosion of an up-to-date mental model which is required for successful intervention. An up-to-date and accurate mental model of the plant's status, called "situation awareness," is considered a defining characteristic of skilled performance in general[8-11]. The mental model is thought to directly drive skill-based processing, control rule-based activity through the mediation of the operator's conscious effort in his working memory and to provide the substantive capability to reason and predict future system states required of knowledge-based processing. Further, a well-developed mental model coupled with accurate situation awareness, enable the operator's performance to be- come more "open- loop" and thus, system control becomes more anticipatory and "smoother" [9] through the use of prediction and expectancy to guide control responses. However, being out of the loop can also make detection of system failures difficult [12]. This problem becomes greater when the operator is largely removed from the control loop as is the case in highly automated systems $[12,13]$.

The shift in roles has other significant effects on the operator as well, such as, a shift from high physical to high cognitive workload (rather than the expected reduction in overall workload) and the potential erosion of the skills to perform the task in the event of automated system failure. Since many advanced NPP designs still require the operator to assume control in the event of a severe transient and to act as the last line of defense, the consequences of poor integration of the operator in the plant design can be quite serious.

The design of the ACR HSI can have important effects on operator performance as well[15]. Computer-based HSI design requires, to a far greater extent than traditional control room designs, the specification of cognitive requirements and processing resources that the operator must utilize in task performance, i.e., cognitive task analysis. Four aspects of HSI are primarily responsible for this requirement. First, information is typically presented in "predigested" form, i.e., raw data parameters are processed and integrated into a higher level of information, thus potentially obscuring their meaning. The variety of ways that data/information can be processed and displayed can be overwhelming. Poorly designed displays will be ignored or, worse, will mislead and/or confuse the operator. Thus the design of the interfaces can have very significant effects on human reliability and system safety yet little is known in the industry about these effects. For example, the main purpose of the safety parameter display system (SPDS) in conventional NPPs is to assist operators at detecting, interpreting, and tracking process disturbances by providing a concise display of key parameters and an ability to track changes. However, problems with the design of SPDS interfaces have been reported[14]. In some SPLS implementations, poor information displays have led to poor acceptance of these potentially valuable systems.

Second, the operator typically has much more infurmation available which, if not properly organized and presented, can be lead to excessively high cognitive workload and be overwhelming. Third, information is typically resident in a "virtual" workstation, rather than in dedicated spatial locations spread out across control stations. Information is located somewhere in a computer system which provides only a glimpse of its contents (through a display device) at any one time. A poorly designed interface can make location of information and navigation through data difficult. Fourth, the flexibility of software-driven interfaces can increase the workload associated with managing the interface and take the operators attention away from the operator's primary task of controlling the process.

In summary, while $A C R$ technology has the potential to improve system performance, the potential also exists to negatively impact human performance and create precursors to 
human error. Those issues most frequently associated with advanced computer-based systems are:

- Poorly organized and ill defined tasks resulting from inadequate allocation of runction strategies,

- Shift from physical to high cognitive workload impairing the operator's ability to monitor and process all relevant data,

- Increase in the operator's cognitive workload associated with managing the interface (e.g., opening, positioning, scaling windows). Managing the interface is irrelevant to the operator's primary task of managing the process and these efforts detract from his primary role in the system,

- Difficulty navigating through and finding important information presented in a computer-based workspace (i.e., information must be accessed through the computer system rather than always present in fixed position annunciators, meters, indicators, chart displays, etc.),

- Loss of operator vigilance due to automated systems resulting in reduced ability to detect off-normal situations,

- Loss of the ability to utilize well-learned, rapid eye scanning patterns and pattern recognition from spatially fixed parameter displays (a particular problem with highly flexible CRT-type interfaces),

- Difficulty understanding how advanced systems works which can lead to either a lack of acceptance of operator aids or an overreliance on them,

- Loss of the operator's "situation awareness" which makes it difficult to assume direct control when appropriate,

- Loss of skill proficiency for the occasional performance of those functions which are typically automated, and

- Great shifts in operator workload (workload transition) in the event of a computer failure.

While human performance issues associated with advanced ACR technology have been identified, they are not necessarily risk significant. The relationship of these effects to human error is of central concern to plant risk evaluations.

ACR technology has been associated with human error as well[3,16,17]. After studying human errors associated with aircraft cockpit automation, Sexton observed that "if the pilot is not provided with enough information with which to make decisions, or decisions are automatically made without providing the rationale to the pilot, the pilot's ability to stay ahead of the aircraft is lost. Complacency and inability to take timely and proper action result "[7]. Rather than eliminating error, increased automation has been associated with a shift of human error to higher functional levels in the system.
Weiner[6] evaluated the result of increased automation in civilian aviation and identified several new categories of error. What is important to an understanding of how such errors arise is a specification of the mechanism by which the performance issues identified above lead to human error. Work on human error models have begun to establish such a link.

Reason[18,19] has presented a model characterizing human error as a predictable result of a tendency to overutilize cognitive processes which serve to simplify complex information tasks. Operators under high workload conditions and lacking the data required to clearly identify appropriate schemata will employ two heuristics called "similarity matching" and "frequency gambling." These heuristics give rise to a number of "basic error tendencies" in human performance which account for many human errors. Similarity matching reflects the tendency for the operator to attempt to match a perceived information pattern (such as a pattern of alarm signals) with an already existing knowledge structure. The operator cognitively tries to establish a link with the stored knowledge structure since it contains a previously identified successful action sequence, thus saving the resource-intensive effort of knowledge-based reasoning. When the perceived information partially activates more than one schema, the discrepancy is resolved by selection of the schema most frequently used in the past. This is the "frequency gambling" heuristic. NPP operators are confronted with what Reason refers to as a "complex multiple-dynamic configuration." The problem configuration changes as a result of both the operators' actions and the system's actions which can create high variability in the problem. This is the most difficult type of problem-solving situation and the one in which beuristics are most likely to be relied on.

While ACR design may impact human performance and human error, the effect of human error on plant safety must be evaluated. The difficulties of quantifying this relationship are addressed in the following section.

\section{IMPLICATIONS FOR HRA}

Under current certification and licensing practices for new plants, the NRC requires a probabilistic risk assessment (PRA) to assure that a pro-josed NPP does not pose undue risk to the public and the environment. A major issue exists in the portion of the PRA addressing human action in advanced technology plants. Since the contributors to unreliability are different from those which are familiar contributors to human error in conventional control rooms, they are less obvious and generally less well understood[20]. Several issues impacting the conduct of HRA for advanced reactors are considered below.

\section{Modeling Human Action in Advanced NPPS}

The diversity of reactor types (such as advanced light water reactors, heavy water reactors, liquid metal reactors, 
and gas-cooled reactors) raises numbers of new issues relative to the design and operation of the reactors. These issues included reactivity control and other reactor physics issues, core thermal hydraulics, natural cooling of the core, very different safety systems and safety system control and operation, smaller plants and multiple units (as many as nine per site), different dominant accident sequences, new hazards (e.g., sodium-water reactions and very high tritium levels), new equipment (liquid sodium pumps, gas circulators, and "passive" components), and advanced instrumentation and controls. These will result in different operator roles and tasks which must be modeled and analyzed.

The effect on human performance of the shift in the operator's role toward supervisory control is also nct well understood (as discussed above). These factors have implications for a wide range of HRA issues including the emergence of new and different types of errors that have not been considered in previous PRAs for "conventional" plants but which must be reflected in the human action modeling in fault and event trees.

\section{Error Quantification}

There are few, if any, human error databases providing data on operator zerformance in supervisory control tasks, operator performance with advanced technology interfaces, or test, mainterance, and calibration tasks with advanced systems. Thus, the formulation of point estimates for base-case human error probabilities (HEPs) will be even more difficult than it already is for conventional operator tasks and the HEPs will have greater uncertainty. One method of addressing this problem is the use of simulators to derive HEPs. This approach has been the subject of an Electric Power Research Institute (EPRI) study[21] and has used in several PRAs[e.g., 22].

Sinulation can be used to collect valuable data on human performance parameters, such as decision-making, that are not well modeled using more traditional HRA approaches. However, the use of simulator-generated data to estimate realworld point estimates is complicated and the generalization from simulation to real-world still contains uncertainty and must be made with caution. Simulator exercises will not reflect the influence of all important performance shaping factors. For exampie, the stress associated with a simulated transient will not be the same as the real-world event. Neither will factors such as control room distractions and noise. The simulated environment alters characteristics of the human information processing system as well, such as initial signal detection, event recognition, and response selection. When a simulator exercise begins, the operator knows something other than normal operations will unfold. During simulated events, very low probability events are likely to occur and the operator expects them - unlike the real world. Underarousal, boredom, and lack of vigilance will not be significant problems on a simulator. The operator's attention is aroused to the initial detection of problems. As the situation develops, events which the operator would never expect to occur in the real world are given a high likelihood; i.e., events with a $10^{-6}$ probability of happening occur several times a day on a simulator. The operator's interpretation of the situation is optimized for failure recognition. For example, in a real control room, an operators initial hypothesis with respect to an unexpected alarm may be that a maintenance activity or miscalibration, etc., is responsible, while in a simulator, it is unlikely these possibilities will be considered. The operator's response to the event will be optimized as well, according to established procedures. There are no job-related consequences to responses made on a simulator, such as poison water injection, that might raise conflicts between safety and productivity. There are major consequences to real-world actions which will affect an operator's probability and timing of taking actions.

This discussion should not be interpreted as indicating that simulator data is not useful. On the contrary, these data are extremely valuable. But, the use of simulator data for realworld point estimation is not well understood at this time, which contributes to uncertainty in its application.

Even when/if these problems can be adequately addressed, there are many additional issues that need to be addressed, such as the amount of data that would be required to develop HEP point estimates within reasonable confidence bands for the various human actions.

\section{Performance Shaping Factors (PSFs)}

ACRs will utilize state-of-the-art technology such as color graphic displays, touch screens, "soft" controls, and expert systems. The human engineering guidelines for the design of advanced HSI is limited, thus it is much less apparent to the HRA analyst whether an ACR design is good and poor. There is also limited understanding of the effects of these technologies on human performance and the nuclear industry has little experience (at least in the U.S.) with operator performance in advanced design NPPs. Thus, it may be difficult to assess the impact of specific design features as performance shaping factors.

\section{Availability of Subject Matter Expertise}

HRA methodology frequently depends on soliciting the judgement of subject matter experts to assist in (1) human action modelling, (2) the determination of the development of base case HEPs, and (3) the evaluation of the importance and quantitative effects of performance shaping factors. However, there are few operations or human factors experts in the area of ACR design and operations available to provide the expertise.

\section{CONCLUSIONS}

The effects of advanced technology on human performance cannot a priori be assumed to be positive. However, the 
conduct of HRA for advanced plants is made difficult by limitations in our general knowledge about the role of human operators in ACRs, the effects of specific design characteristics on human performance, the lack of available databases, and the lack of available subject matter expertise. Thus, at present there is a heightened need to precisely and thoroughly document the process of human action identification, evaluation, and quantification. In the longer term, research is needed on the effects of advanced technology on human performance including the assessment of industry experience on a world-wide basis. The development of models to better understand the cognitive underpinnings of human error appears to be an especially promising path. While the use of simulators may facilitate our understanding of human functioning in advanced control rooms, its use for point estimation must be further developed.

\section{REFERENCES}

[1] W. Kennedy, "Survey of OECD members on the use of computers in control rooms of Nuclear power plants," in Man-machine interface in the nuclear industry, Vienna, Austria, 1988.

[2] R. Carter and R. Uhrig, "Human factors issues associated with advanced instrumentation and controls technologies in nuclear plants," Washington, D.C.: U.S. Nuclear Regulatory Commission, NUREG/CR-5439, 1989.

[3] A. Coblentz, "Vigilance and performance in automated systems," NATO ASI Series D, Vol. 49, 1988

[4] N. Moray and B. Huey (eds.), "Human Factors Research and Nuclear Safety," Washington, D.C.: National Research Council, National Academy of Sciences, 1988.

[5] R. Pew et al., "Research Needs for Human Factors," Washington, D.C.: National Research Council, National Academy uf Sciences, 1983

[6] E. Wiener and R. Curry, "Flight-deck Automation: Promises and Problems," Ergonomics, Vol. 23, pp. 995-1011, 1980.

[7] G. Sexton, "Cockpit-Crew Systems Design and Integration," in E. Wiener and D. Nagel (eds.), Human Factors in Aviation, Academic Press, pp. 495-525, 1988.

[8] C. Wickens, Engineering Psychology and Human Performance, Columbus, OH: Merrill Publishing Company, 1984.

[9] N. Moray, P. Lootsteen, and J. Pajak, "Acquisition of Process Control Skills," IEEE Transactions on Systems, Man, and Cybernetics, Vol. 16, pp. 497-504, 1986.

[10] J. Rasmussen, "Skills, Rules, Knowledge: Signal, Signs, and Symbols and Other Distinctions in Human Performance Models," IEEE Transactions on Systems, Man, and Cyhernetics, Vol. 13, pp. 257-267, 1983
[11] T. Sheridan, "A General Model of Supervisory Control," in T. Sheridan, and G. Johannsen (eds.), Monisoring Behavior and Supervisory Control, New York, NY: Plenum Press, 1976.

[12] C. Wickens and C. Kessel, "The Detection of Dynamic System Failures," in J. Rasmussen and W. Rouse (eds.), Human Detection and Diagnosis of System Failures, New York, NY: Plenum Tress, 1981.

[13] A. Ephrath and L. Young, "Monitoring vs. Man-In-The-Loop Detection of Aircraft Control Failures," in J. Rasmussen and W. Rouse (eds.), Human Detection and Diagnosis of System Failures, New York, NY: Plenum Press, 1981.

[14] R. Liner and J. DeBor, "Progress Reviews of Six Safety Parameter Display Systems," Washington, D.C.: U.S. Nuclear Regulatory Commission, NUREG/CR-4797, 1986.

[15] J. Patrick, "Information at the human-machine interface," In J. Rasmussen, K. Duncan, and J. Leplat (eds.), New technology and human error, New York, NY: J. Wiley and Sons, Publishers, 1987.

[16] J. Rasmussen, K. Duncan, and J. Leplat (eds.), New technology and human error, New York, NY: J. Wiley and Sons, Publishers, 1987.

[17] E. Wiener and D. Nagel (eds.), Human factors in aviation, New York, NY: Academic Press, 1988.

[18] J. Reason, "Generic error-modelling systems (GEMS): A cognitive framework for locating common human error forms," in J. Rasmussen, K. Duncan, and J. Leplat (eds.), New Technology and Human Error, New York, NY: J. Wiley and Sons, 1987.

[19] J. Reason, "Cognitive aids in process environments: prostheses or tools?" in E. Hollnagel, G. Mancini, D.D. Woods (eds.), Cognitive Engineering in Complex Dynamic Worlds, New York, NY: Academic Press, 1988.

[20] J. O'Hara and R. Hall, "Human-computer interface and human reliability," Proceedings on advances in human factors research on man/computer interactions, Nashville, TN: American Nuclear Society, 1990, pp. 339-345.

[21] A. Spurgin :i al., Operator Reliability Experiments Using Power Plant Simulators, Palo Alto, CA: Electric Power Research Institute, EPRI NP-6937, 1990.

[22] L. Weston, et al., Recovery Actions in PRA for the Risk Methods Integration and Evaluation Program, Washington, D.C.: U.S. Nuclear Regulatory Commission, NUREG/CR4834), 1987 

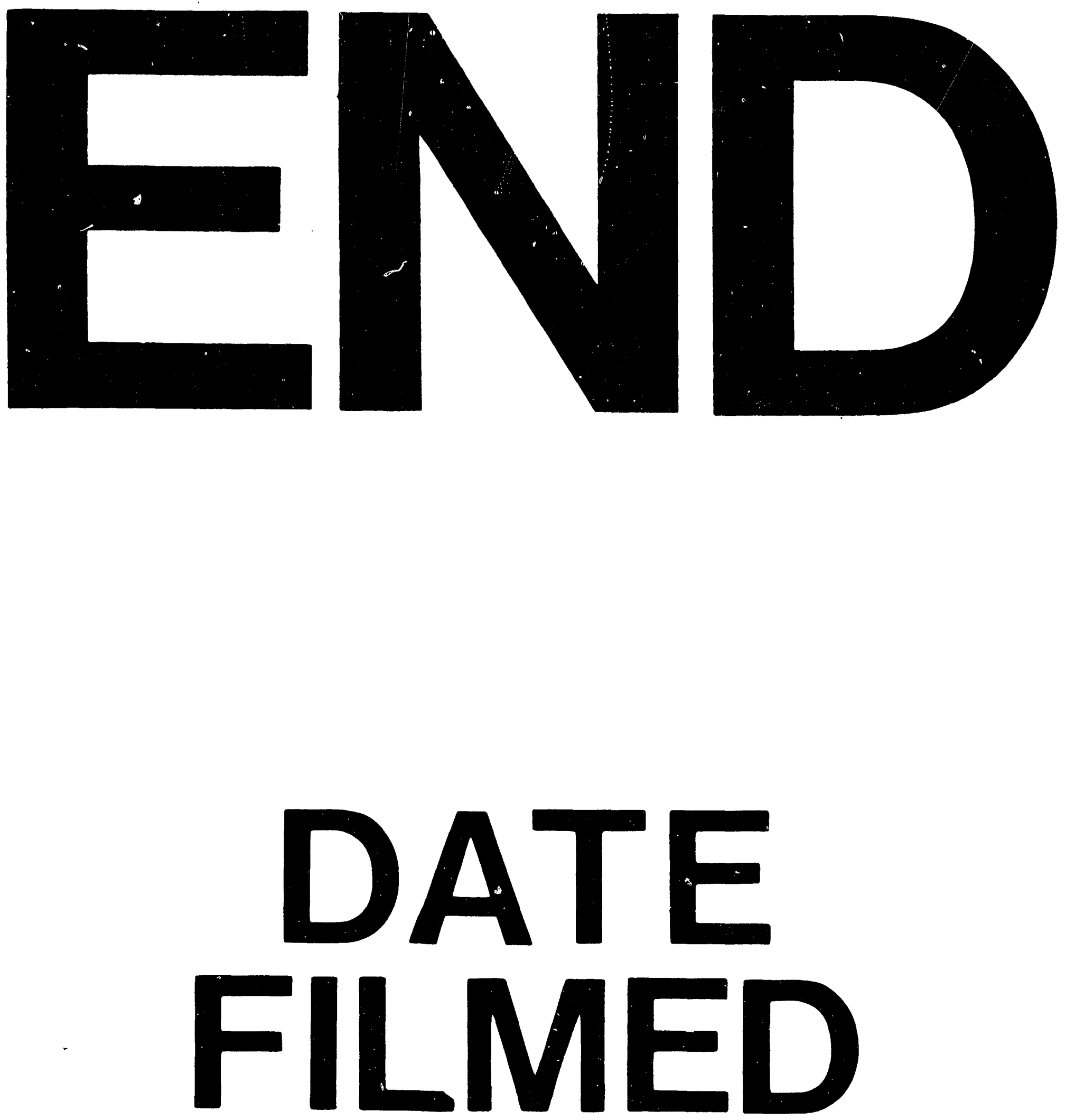

1

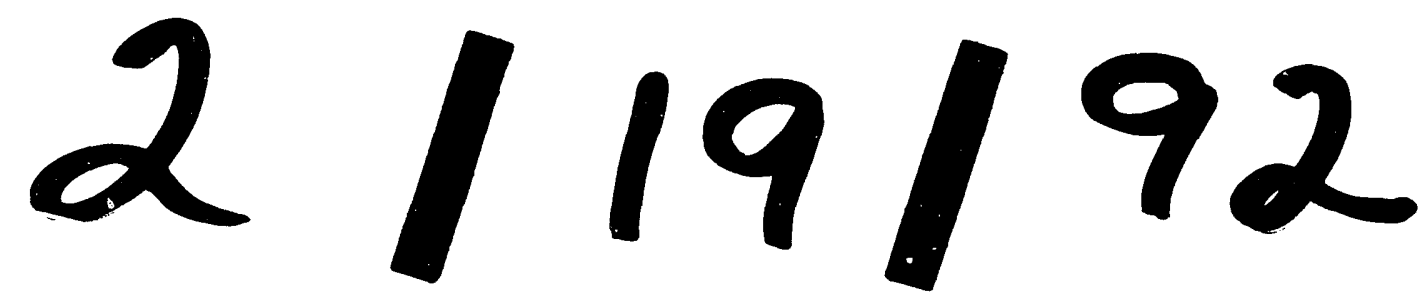


